\title{
Antioxidant properties of fresh, powder, and fiber products of mango (Mangifera
} foetida) fruit.

\begin{abstract}
This study was aimed to investigate the antioxidant capacity and antioxidant components in fresh, powder and fiber products prepared from Mangifera foetida fruit, locally known as bacang. The antioxidant capacity and components (reducing, flavonoid, carotenoid, and ascorbic acid contents) were in the order of fresh $>$ fiber $>$ powder, whereas based on $\beta$ carotene bleaching method, order of antioxidant activity was fiber $>$ powder $>$ fresh. Pearson correlation coefficient showed that there was positive and significant correlation $(r>0.9)$ between antioxidant capacity and certain antioxidant components.
\end{abstract}

Keyword: Antioxidant capacity; Antioxidant components; Mangifera foetida 